\title{
Adaptive Model based on Proactive Spectrum Sensing for Emergency Cognitive Ad hoc Networks
}

\author{
Sasirekha GVK \\ International Institute of Information Technology \\ Bangalore, India \\ sasirekha@iiitb.ac.in
}

\author{
Jyotsna Bapat \\ International Institute of Information Technology \\ Bangalore, India \\ jbapat@iiitb.ac.in
}

\begin{abstract}
Emergency Cognitive Ad hoc Networks (ECANs) require proactive, adaptive and resource efficient spectrum sensing. In general, work in the area of spectrum sensing for cognitive radio networks, has been focused on improving the performance and resource efficiency in static models based on reactive sensing. In this paper, an adaptive model based on proactive spectrum sensing is proposed for ECANs that have dynamic number of users and varying signal to noise ratios. The proposed model uses collaborative spectrum sensing scheme, where each cognitive radio senses the spectrum with certain probability. A Spectrum Coordinator (SC) plays the role of fusion center, generating a fused group decision based on the sensed information obtained from SUs in the network. SC allocates a 'reward' to each SU proportional to its sensing effort and the targeted performance. It can be seen that fairness in energy consumption across SUs is achieved in the proposed scheme.
\end{abstract}

Keywords- Spectrum sensing; Cognitive adhoc networks; Proactive sensing; Bayesian risk; Resource efficiency

\section{INTRODUCTION}

Emergency Cognitive Ad hoc Networks (ECANs) are infrastructure-less networks formed within a short span of time, having time-varying number of users experiencing varying Signal to Noise Ratios (SNRs). They find applications in disaster management, military and other emergency situations. Adaptive Ad hoc Freeband communications (AAF) project [1] is such example, where the ideas of cognitive radio are applied to the field of public safety and emergency control communications. Sensing the spectrum to identify its occupancy by the licensed Primary Users (PUs) is a crucial functionality of any cognitive radio network. Cognitive radios, also referred to as Secondary Users (SUs) are involved in identifying the unused licensed spectrum. Sensing of spectrum may be performed either reactively or proactively. Reactive sensing is done 'on request' when that particular frequency is planned to be used. Though reactive sensing gives more current information on the spectral occupancy, it tends to add latencies in the operation, especially when the sensing time is longer [2]. Spectrum sensing can be performed in a standalone manner by each SU independently or can be done in a collaborative way [3]. In collaborative spectrum sensing, the local sensing information from each SU is received by the fusion center, which fuses this information and generates a group decision. Latencies of reactive sensing become more pronounced when collaborative spectrum sensing is applied because of the above mentioned processing involved.
ECANs require the sensing models to meet the targeted sensing accuracy, resource efficiency and low latency in the delivery of packets. They also need to be adaptive to varying number of SUs and SNR conditions. Since the radios are battery operated, fairness in energy consumption is also an important factor. Only few SUs sensing most of the time could lead to the depletion of their energies and decrease their life in the network. For a longer sustenance of the network, it is necessary that the sensing load should be distributed uniformly across all SUs. Data fusion technique applied to ECANs needs to be resilient to data falsification attacks (Byzantine attacks) [4]. In such attacks malicious users send false sensing data to the fusion center leading to increased probability of incorrect sensing. 'OR'ing is a technique used commonly for data fusion because of its simplicity [3]. However, the technique is prone to data falsification attacks. Likelihood Ratio Test (LRT) based techniques are more resilient and are thus more suitable for the ECANs. The performance superiority of LRT based fusion in this respect is shown in [5].

Literature survey shows that there is no existing model to cater for all the above requirements of ECANs. Most of the earlier work done on collaborative spectrum sensing has been focused on the performance and resource optimization for static situations and reactive schemes that may not be suitable for ECANs. For example, [6] discusses the sensing parameter optimization w.r.t. the number of cooperating users. Optimization of sensing throughput tradeoff has been presented in [7]. [8] Shows that it will suffice if an optimal subset of SUs sense the spectrum in a given time epoch resulting in resource efficiency without loss of spectral information.

This paper presents a new proactive adaptive model, where the spectral band is periodically sensed by the SUs in the synchronized quiet period. Discussion about the quiet periods can be found in [9]. The frequency as well as duration of these quiet periods is adapted according to the PU behavior as discussed in [10]. The proposed model is adaptive to the changes in the network size and SNR conditions. The analytical model and simulation results confirm that the targeted sensing performance can be met with minimal number of SUs sensing in each epoch and with fairness in energy consumption. The proposed adaptive model meets all the requirements using collaborative spectrum sensing scheme, where each SU senses with certain probability of sensing $\left(P_{s}\right)$ and a spectrum coordinator receives sensing information from the different SUs in the network and generates a 'fused decision' based on LRT (Refer Figure 1). 


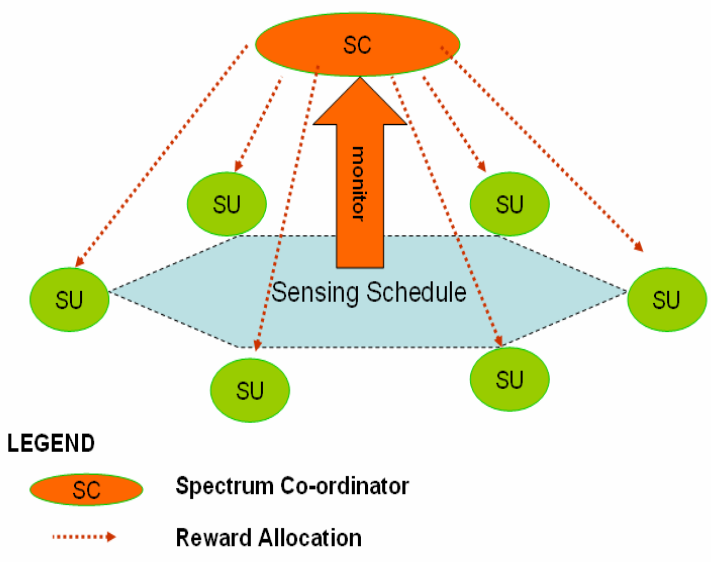

Figure 1. Cognitive Adhoc Network Scenario

The rest of the paper is organized as follows: Section II describes the system model; Section III gives the analytical model; and Section IV discusses the proposed adaptive system model and its ability to adapt to the varying network size and SNR conditions. Section V presents the simulation results. Section VI contains the discussions on the proposal. Finally the conclusions and future work are presented in section VII.

\section{SYSTEM MODEL}

Consider an ECAN with a collaborative spectrum sensing mechanism and a chosen SC as shown in figure 1. The network size could be varying from a small values to medium sized one (i.e. less than 100). The Primary Users (PUs) are the licensed users operating in a subset of these frequencies $\left[f_{1}, f_{2}, \ldots . f_{r}\right]$. It is assumed that the distance between the secondary users or sensors is much smaller compared to their distance from the PU. As a result, the measurements from the SUs are essentially correlated. The SUs however, experience independent and identically distributed fading channels [11]. Each SU makes independent measurement of the spectral information and the individual local decisions about spectral occupancy are sent to $\mathrm{SC}$ for data fusion.

Common Control Channel: A common error-free control channel is used to communicate the local decisions from each SU across the network to the SC [12].

Mobility Model: Mobility can be classified as a "nomadic community" model. In this model, a group of mobile nodes move from one position to another. Such models are beneficial in real life emergency applications such as disaster management and military. Restricted movement among the nodes is required when group travels from one location to another. In this model, reference point of each node is determined based on the general movement of the group. The SNRs of the SUs are assumed to be exponentially distributed with an average $\gamma$, referred to as the group SNR. This network is associated with an average probability of detect $P_{d}$ and probability of false alarm $P_{f}$ corresponding to the group SNR $\gamma$ [13].
Spectrum Sensing details: Each Secondary User (SU) senses a set of $r$ spectral bands centered at frequencies $\left[f_{1}, f 2, \ldots . f_{r}\right]$ using energy detection. The threshold is set for a desired probability of false alarm and the estimated noise power. The SUs sense a given set of frequencies proactively in the quiet period of the network. The quiet periods are synchronized across the network. Though difficult, time synchronization is indispensable is CAN due to the requirements of coordinated and simultaneous quiet periods for spectrum sensing, as well as the common understanding of time frame/slot in many CR MAC designs. CR-Sync protocol has been proposed in [14], that achieves network-wide time synchronization in a fully distributed manner. The local spectrum sensing could be based on simple energy detection, matched filter, cyclostationarity or any other proven method [3].

PU usage pattern: The usage pattern could be of any distribution which is known prior and can be modeled using a 2 stage Markov model [10].

Data Fusion: LRT based fusion rule is considered in the model. The probability of detect and probability of false alarm of each SU required for LRT may be estimated by the spectrum coordinator using the counting rule [15 \& 23]. Any other fusion technique like 'AND', 'OR' or Majority Logic may also be used instead [3]. The objective of the SC is to provide communication within the network while ensuring minimal interference to the primary users. In order to achieve this objective, the spectrum coordinator performs the following functions. a) Data Fusion b) Monitoring and estimating the sensing probability, probability of detect and probability of false alarm of each SU c) Allocate reward to each SU based on the sensing probability estimated and the targeted sensing performance. This is discussed further in section IV.

\section{ANALYTICAL MODEL}

For the system model described in Section II, the analytical model is derived in this section.

Let $R_{k}$ be the Bayesian risk factor defined in terms of probability of detection of the fused decision $\left(Q_{d k}\right)$ and the probability of false alarm of the fused decision $\left(Q_{f k}\right)$ as given in [17]; where $k$ is the number of inputs fused using LRT obtained from $k$ sensing SUs.

$$
R_{k}=C_{F} Q_{f k}-C_{D} Q_{d k}+C
$$

Where

$$
\begin{aligned}
& C_{F}=P_{0}\left(C_{10}-C_{00}\right) \\
& C_{D}=\left(1-P_{0}\right)\left(C_{01}-C_{11}\right) \\
& C=C_{01}\left(1-P_{0}\right)+C_{00} P_{0}
\end{aligned}
$$

$P_{0}$ is the Probability of the PU being off; $C_{00}, C_{01}, C_{10}$ and $C_{11}$ are the costs associated with making the right and wrong decisions [17]. $P_{d}$ and $P_{f}$ denote the average values of the probability of detection and probability of false alarm of the network as a whole, related to the combined or fused decision. The notation of the various terms is tabulated in Table I. 
TABLE I: TERMinOLOGY TABLE

\begin{tabular}{|c|c|}
\hline Notation & Description \\
\hline$k$ & Number of sensing SUs in a given time epoch \\
\hline$\gamma$ & Group SNR \\
\hline$P_{d}$ & Average probability of detect of the group \\
\hline$P_{f}$ & Average probability of false alarm of the group \\
\hline$N$ & Network size (integer) \\
\hline$Q_{d k}$ & $\begin{array}{l}\text { Probability of detect of fused data obtained by } \\
\text { fusing k inputs }\end{array}$ \\
\hline$Q_{f k}$ & $\begin{array}{l}\text { Probability of false alarm of fused data obtained } \\
\text { by fusing k inputs }\end{array}$ \\
\hline$m$ & $\begin{array}{l}\text { Lower limit of summation of (3) and (4) as given } \\
\text { in [17] }\end{array}$ \\
\hline$P_{0}$ & Probability of PU being in off state \\
\hline$M$ & $\begin{array}{l}\text { Observation interval as an integer number of time } \\
\text { epochs }\end{array}$ \\
\hline$R_{k}$ & Risk Factor [17] \\
\hline$R_{k t}$ & Risk Factor in each time epoch $\mathrm{t}$ \\
\hline$K$ & $\begin{array}{l}\text { Minimal Number of SUs required to maximize } \\
\text { the network utility } J_{k}\end{array}$ \\
\hline$P_{s_{-} o p t}$ & Desired probability of sense equal to $K / N$ \\
\hline$P_{s_{-} a v}$ & Average probability of sense in $M$ time epochs \\
\hline$J_{k}$ & Network utility function in terms of $k$ \\
\hline$J_{P s \_a v}$ & $\begin{array}{l}\text { Network utility function in terms of average } \\
\text { probability of sense }\end{array}$ \\
\hline$\eta_{k}$ & Resource efficiency \\
\hline$I_{k}$ & Certainty factor $=1-$ risk factor \\
\hline$I_{P s_{-} a v}$ & $\begin{array}{l}\text { Certainty factor in terms of average probability of } \\
\text { sense }\end{array}$ \\
\hline$\alpha$ & $\begin{array}{l}\text { the weightage given to the accuracy and resource } \\
\text { efficiency } \eta_{k} \text {. }\end{array}$ \\
\hline
\end{tabular}

$Q_{d}(k)=\sum_{i=m}^{k}\left(\begin{array}{c}k \\ i\end{array}\right) P_{d}^{i}\left(1-P_{d}\right)^{k-i}$

$Q_{f}(k)=\sum_{i=m}^{k}\left(\begin{array}{l}k \\ i\end{array}\right) P_{f}^{i}\left(1-P_{f}\right)^{k-i}$

Where $m$ is the number of users that need to be in agreement to make a decision about presence or absence of PU, as described in detail in [17]. Certainty Factor (CF) defined below is used to define the Network utility function similar to one in [16]

$I_{k}=1-R_{k}$

$J_{k}=\alpha I_{k}+(1-\alpha) \eta_{k}$

$0 \leq \alpha \leq 1, \eta_{k}=\frac{N-k}{N}$

Where $N$ is the network size and $N$ is expected to be timevarying with new SUs joining as well as leaving the network. $k$ is the number of sensing SUs, which again may vary from epoch to epoch. It is assumed that change in value of $N$ is updated with a frequency lesser than that of $1 / M$ time epochs, where $M$ time epochs is the observation period. The weighting factor $\alpha$ signifies the weightage given to the accuracy and resource efficiency $\eta_{k}$.

When averaged over $M$ time epochs, CF can be expressed as a function of average probability of sense $P_{s}{ }_{a v}$, instead of the number of sensing SUs. With each SU sensing with average probability $P_{s_{-} a v}, \mathrm{k}$ can be approximated as $k=P_{s_{-} a v} N$.

$I_{P_{s_{-} a v}}=1-\frac{1}{M} \sum_{t=1}^{M} R_{k_{t}}$

Equation (5) can be rewritten as,

$$
J_{P_{S_{-} a v}}=\alpha I_{P_{s_{-} a v}}+(1-\alpha)\left(1-P_{s_{-} a v}\right)
$$

As the average probability of sensing $P_{s_{-} a v}$ increases, the $\mathrm{CF}$ improves, while the network efficiency decreases and vice versa. Let us say, the maximum of the utility function occurs some $P_{\text {sopt }}=K / N$. The average probability of sense corresponding to the maximum is what a network should adapt and converge to.

Location of this maximum depends upon values of $\alpha, \gamma, N$. Figure 2 depicts the relationship between optimum $P_{s_{-} a v}$ that will maximize the network utility and $\alpha, N$ and average $P_{d}$. As $\alpha$ increases, the weightage given to resource utilization decreases, as a result, the required sensing probability is higher. With increasing $N$ the sensing probability to achieve the maximum decreases as the sensing effort for the same information gain required to be put in by each $\mathrm{SU}$ reduces. Increasing $P_{d}$ (for constant $P_{f}$ ) or decreasing $P_{f}$ (for constant $P_{d}$ ) means improved SNR. Increase in average SNR reduces the sensing effort required by each SU for a targeted sensing performance. It should be noted that $Q_{d k}, Q_{f k}, R_{k}$ and $I_{k}$ depict various metrics for measuring the sensing performance. They are related as expressed in (2), (3), (4) \& (5).

Having derived the analytical model for the network utility function, the following section discusses how to achieve maximum utility adaptively with minimal resource consumption while maintaining the targeted information gain.

\section{ADAPTIVE SYSTEM}

In order to achieve the maximum utility for a given $N$ and SNR conditions, a feedback mechanism is required between the $\mathrm{SC}$ and the SUs. This feedback is provided by a 'reward' allocated to each SU by the SC, which could be in the form of bandwidth, priority in scheduling [18], billing concession [19] or just a factor used for adaptation. Light handed methods of introducing such incentives without imposing too much of overhead have been discussed in depth in [19]. How to built systems that can incentivize radios to follow the spectrum rules has been discussed in [19]. This motivates the proposed adaptive system. The reward is proportional to the sensing effort and the targeted performance measured as $Q_{d \text { target }}$ for a constant $Q_{f}$. This metric was chosen for its simplicity and can be replaced by any of the other mentioned sensing performance metrics. The sensing effort of the ith SU is measured as 

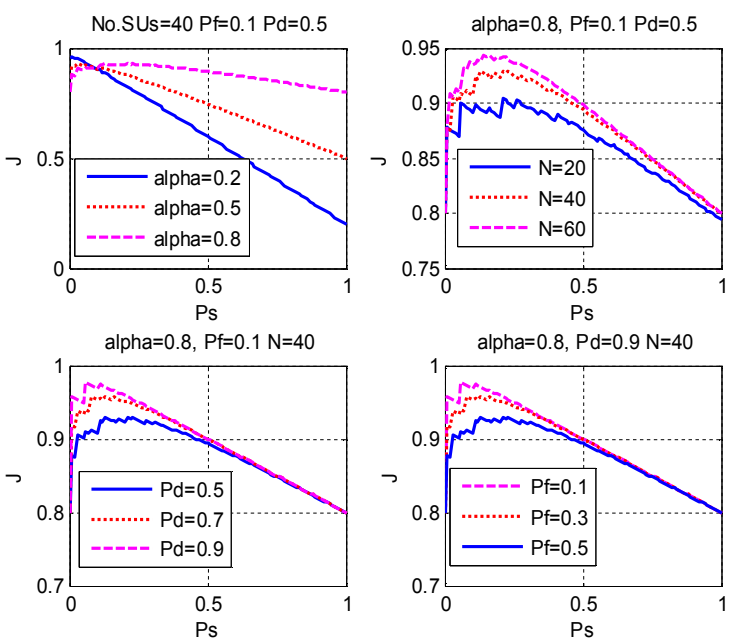

Figure 2. $J_{P_{-} a v}$ versus $P_{s_{-} a v}$ for constant $N$ and different $\alpha \mathrm{s}$

$\hat{P_{s i}}=\frac{s_{i}}{M}$

where $s_{i}$ is the number of times an SU senses in $M$ epochs of the observation interval which is the estimate of probability of sense of each SU $i$. The sensing effort required to achieve the targeted accuracy is given by $P_{s \text { opt }}$ which should be learnt and adapted to by each SU based on events tabulated in Table II. These events encompass the different situations that arise in ECANs with changing number of users and SNRs.

Figure 3 shows the working of the adaptation scheme. The operations of data fusion, estimation of the parameters $\left[P_{d i}, P_{f i}\right.$ ,$\left.P_{s i}\right]$ for each SU and learning of $K$ (optimum number of sensing SUs for the given situation) are performed at the SC. $P_{S_{-} o p t}$ is computed as $K / N$. It is compared with the sensing probability estimate $P_{s i}$ of each SU. The normalized reward $F_{s i}$ is computed for each SU as $P_{s i} / P_{s o p t}$. This reward factor $F_{s i}$ is sent in real time to each SU. Each SU adapts its sensing probability according to the observed reward. If reward is less than 1, it implies that the sensing probability needs to be improved. If reward is 1 then the sensing probability can be reduced.

The update equation for probability of sense when $F_{s i}$ is less than 1 is as shown below.

$P_{s_{-} \text {new }}=P_{s i}+\Delta P_{s i}$

where

$\Delta P_{s i}=\mu_{p}\left(1-F_{s i}\right) / P_{s i} \quad$ where

$0 \leq \mu_{p} \leq\left(1-P_{s i}\right) P_{s i} /\left(1-F_{s i}\right)$

This equation shows that the change in $P_{s i}$ is directly proportional to the difference $\left(1-F_{s i}\right)$. Correction will be higher for smaller values of $F_{s i}$ and vice versa.

The update equation for $F_{s i}$ equal to 1 is shown below. In this case, the update only depends upon the current value of $P_{s i}$. Higher the original $P_{s i}$, higher is the update and vice versa.

$$
P_{s_{-} \text {new }}=P_{s i}-\Delta P_{s i}
$$

$\Delta P_{s i}=\mu_{n} P_{s i}$

$0 \leq \mu_{n} \leq 1-\left(1 / P_{s i}\right)$

The adaptation algorithm at the SC and SU is listed below:

\section{Adaptation Algorithm at SC}

Step 1: Init $k=1$, Set $M$

Qd_target $=0.9 / /$ can be set to any targeted value Ps_opt $=1$

TABLE II: EVENTS FOR TRIGGERING ADAPTATION

\begin{tabular}{|l|l|c|}
\hline Sl. No. & \multicolumn{1}{|c|}{ Events } & Action \\
\hline 1. & Network Startup & $\begin{array}{c}\text { Learn } P_{s_{-} \text {opt }} \text { for given } N \text { and SNR } \\
\text { conditions }\end{array}$ \\
\hline 2. & N changes & Update $P_{s_{-} \text {opt }} \leftarrow \mathrm{N}_{\text {old }} * P_{s_{-} o p t} / \mathrm{N}$ \\
\hline 3. & SNR changes & $\begin{array}{c}\text { Learn } P_{s_{-} o p t} \text { for given } N \text { and SNR } \\
\text { conditions }\end{array}$ \\
\hline
\end{tabular}

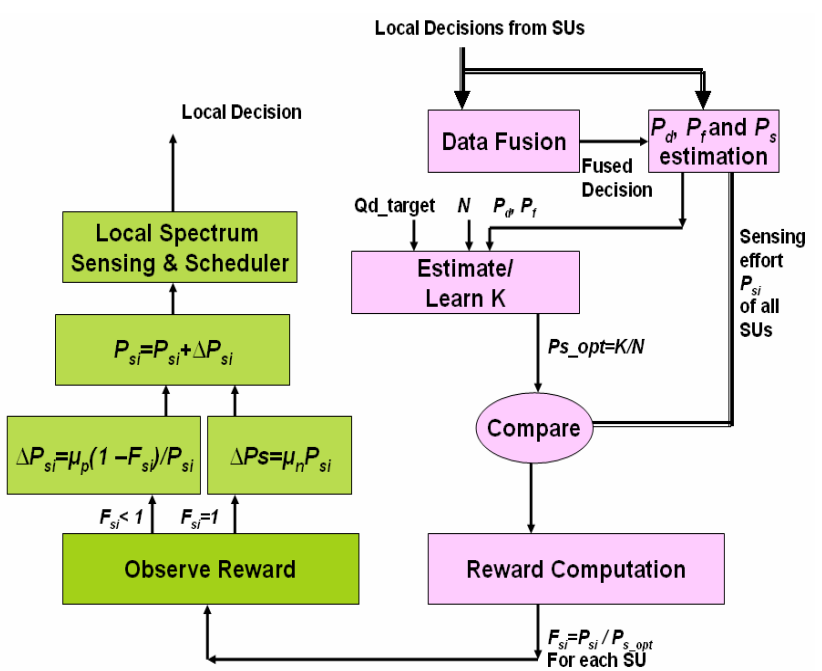

Legend $\square$ At each SU $\square$ At SC

Figure 3: The block diagram of the adaptive system

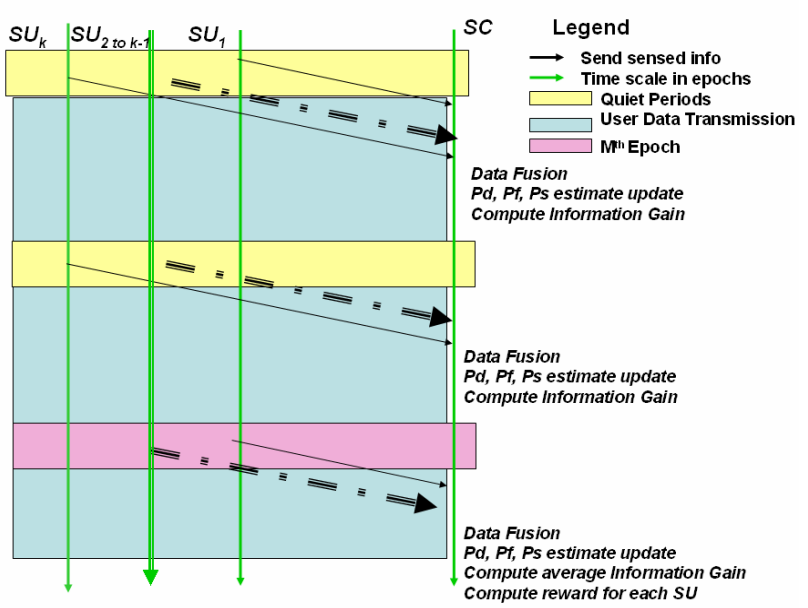

Figure 4: Event Schedule of the adaptive system 
Ps opt Learning flag $=0$

Step 2: Get local decisions

Data Fusion

Update $P_{s i}, P_{d i}, P_{f i}$

Compute Qd

Step 3: if iter $=M$ Compute reward for each $S U$

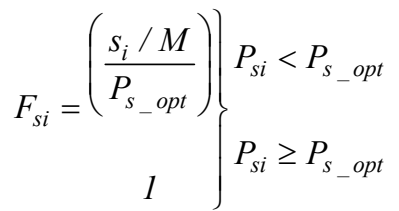

Set iter $=1$

Compute $Q_{d \text { av }}$ (Average $Q_{d}$ over $M$ iters)

If $Q_{d_{-} a v}>Q_{d_{-} \text {target }}$ \& If $P_{s_{-} \text {opt_Learning_flag }}=0$

set $P_{s_{-} \text {opt }} \leftarrow$ mean $\left(P_{s i}\right)$ of all $S U$,

set $P_{S_{-} \text {opt_Learning flag }=1}$

Step 4: iter $\leftarrow$ iter +1

go to Step 2

Step 5: If event 2 set $N \_$old $\leftarrow N, P_{s_{-} \text {opt }} \leftarrow P_{s_{-} \text {opt }} * N_{-}$old $/ N$

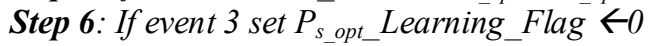

\section{Adaptation Algorithm at the $i^{\text {th }} \mathrm{SU}$}

Step 1:Init: Set M;

Set $P_{s i}=$ Random value $\{0$ to 1$\}$;

$F_{s i}=0 ; \mu_{p}=0.02 ; \mu_{n}=0.2$

Step 2: Energy Detection collecting $m$ samples [3]

Step 3: Make local decisions and send to $S C$

Step 4: if iter $=M$, observe $F_{s i}$

Step 5:If $F_{s i}<1$ and $P_{s i}<1$ set $P_{s i}=P_{s i}+\Delta P_{s i}$

else $P_{s i}=P_{s i}-\Delta P_{s i}$

go to Step 2

Figure 4 shows the scheduling of events of the adaptive system. The SUs sense the spectrum in the quiet period and send the sensing information to the $\mathrm{SC}$, which fuses the data.

\section{Simulation Model and Results}

The simulation model was built using Netlogo [20] to validate the performance of the adaptive system. The parameters considered for the simulation are as listed in Table III. Each of the SUs has identical code but for one SU which is allotted the role of SC. This choice of SC can be done based on the sequence of entry into the network, i.e. first SU that starts the ad hoc network could be the SC. Choice of SC could also be based on hierarchy of ranks or could be dynamic depending on the application. It should be noted that the choice of SC would not affect the simulation results.

Figure 5 shows the variance of the energy spent by each SU and the sensing performance $Q_{d \_a v}$ versus number of iterations (time epochs). The convergence is towards a fair system where all SUs put in equal effort towards sensing, while maintaining targeted $Q_{d}$, i.e. $Q_{d_{-} \text {target }}$. The group $\mathrm{SNR}$ was set to $0 \mathrm{~dB}$ and counting rule was used to estimate $P_{d}\left(P_{f}=0.1\right)$. Figure 5 alsoshows an SU initializing with a random probability of sensing converging to $P_{s \text { opt }}$ learnt by the SC (event 1 of Table
TABLE III: LiST OF PARAMETERS FOR SIMULATION.

\begin{tabular}{|l|l|c|}
\hline Parameter/Scheme & \multicolumn{1}{|c|}{ Value/Type } & \multicolumn{1}{c|}{ Comments } \\
\hline $\begin{array}{l}\text { Local Spectrum } \\
\text { Sensing }\end{array}$ & $\begin{array}{l}\text { Energy } \\
\text { Detection }\end{array}$ & $\begin{array}{c}\text { Can be replaced by any other } \\
\text { local sensing mechanism }\end{array}$ \\
\hline $\begin{array}{l}\text { Number of Samples } \\
m\end{array}$ & 100 & $\begin{array}{c}\text { Can be varied and decides the } \\
\text { accuracy of ED }\end{array}$ \\
\hline $\begin{array}{l}\text { SNR Distribution } \\
\text { across SUs }\end{array}$ & 10 & $\begin{array}{c}\text { Exponential distributed with } \\
\text { different average values }\end{array}$ \\
\hline $\begin{array}{l}\text { Initial Number of } \\
\text { SUs }\end{array}$ & 0.02 & $\begin{array}{c}\text { Considering medium sized } \\
\text { networks with N between 3 to } \\
\text { 50 SUs }\end{array}$ \\
\hline$\mu_{\mathrm{p}}$ & 0.2 & Adjusted Empirically \\
\hline$\mu_{\mathrm{n}}$ & \multicolumn{2}{|c|}{ Adjusted Empirically } \\
\hline
\end{tabular}

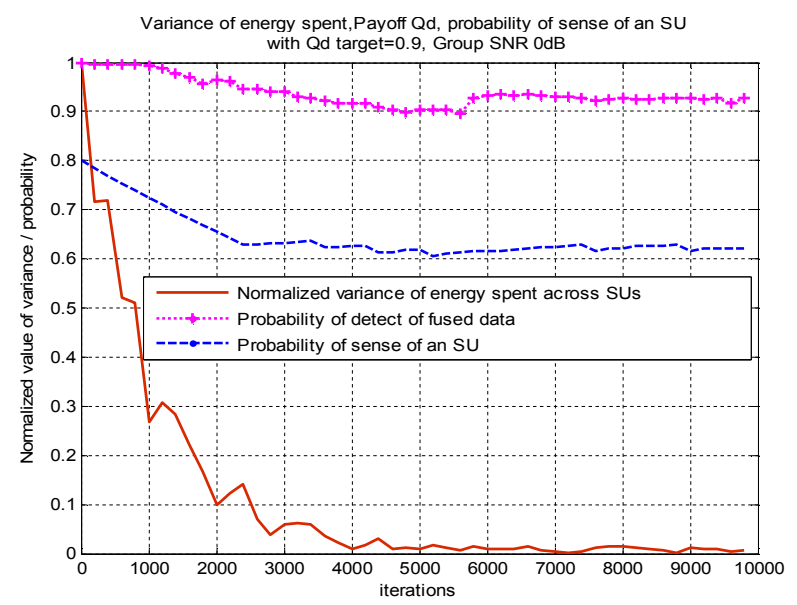

Figure 5:The convergence of the variance of energy spent across SUs while maintaining performance

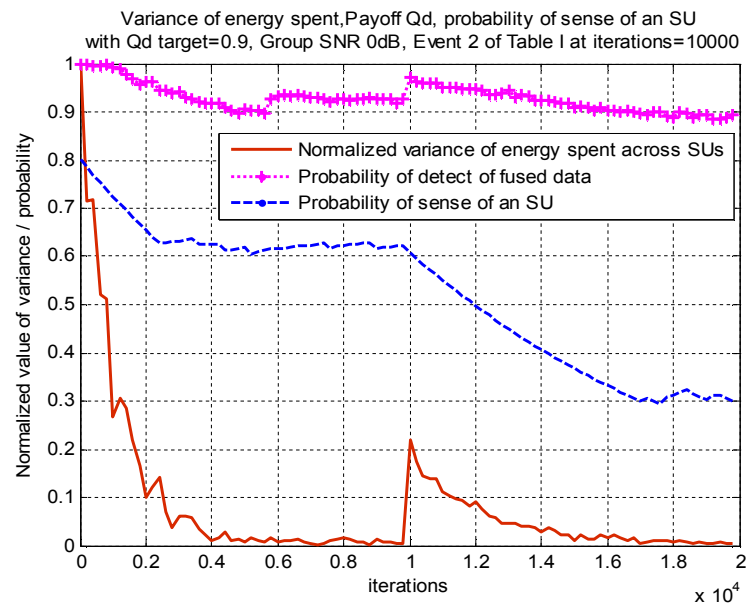

Figure 6:The convergence of the variance of energy spent across SUs, $P_{s}$ at an SU to $P_{s_{-} o p t}$ with number of users N changing at $10000^{\text {th }}$ time epoch

II). Figure 6 shows the variance of the energy spent by each $\mathrm{SU}$, the sensing performance $Q_{d_{-} a v}$ and convergence of probability of sense of an SU when event 2 of Table II occurs at the $10000^{\text {th }}$ time epoch, i.e. new SUs are added to the network. Then the sensing probability is reduced to the updated 


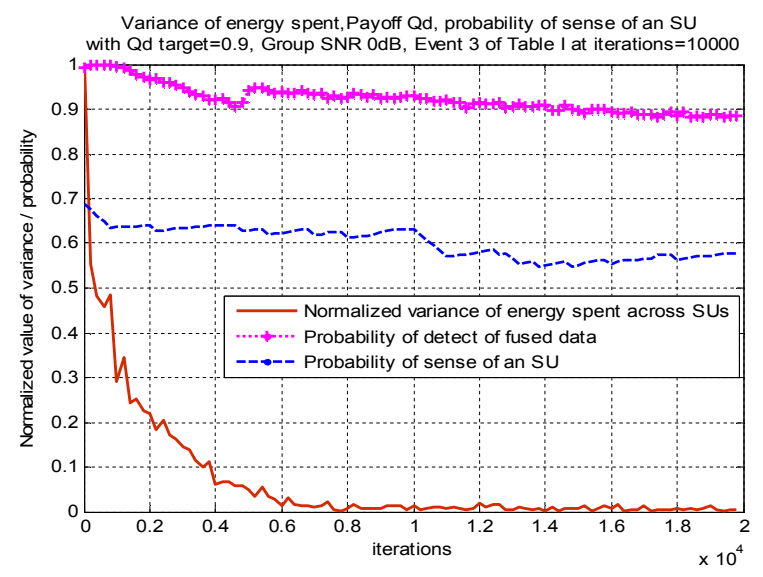

Figure 7: The convergence of the variance of energy spent across SUs and the probability of sense of an SU while maintaining performance with SNR changing at $10000^{\text {th }}$ time epoch

$P_{s \_ \text {opt }}$. Similarly, occurrence of event 3 , i.e. change in SNR values is shown in Figure 7. SNR changes do not cause any abrupt changes in the variance but the $P_{s i}$ of the SUs is adapted to the required new value. This interval time between changes in SNR depends on the channel fading conditions, which in turn depends on the speed of the nomadic group. The model proposed is independent of this parameter. The changes in SNR can occur at any time period that is greater than $M$.

\section{DISCUSSION}

Having observed the convergence of the system under various conditions, an analysis of the convergence time is discussed in this section. The convergence time is directly proportional to the observation time period $M$ which is measured in epochs of time between quiet periods. This quiet period can be determined based on the PU usage pattern [10]. The convergence time also depends on the amount of adaptation and the size of the adaptation step size.

\section{CONCLUSIONS}

An adaptive model for collaborative spectrum sensing in ECAN was developed. The analytical and simulations models were presented. The model supports dynamic number of SUs as well as dynamic SNR conditions. The sensing is done by SUs in the quiet periods which are synchronized. Each SU senses with a probability of sense which is adapted based on the feedback from the SC provided in the form of reward. Results showing the convergence of the adaptation towards a resource efficient condition while maintaining the sensing performance and fairness. The SUs of the CAN are assumed to be embedded systems which are not been tampered. Attacks against cognitive radio networks have been well addressed in $[21,22]$. The model presented is relatively resilient to data falsification attacks because of the LRT based data fusion.

\section{REFERENCES}

[1] F. Brouwer, M. de Graaf, H. Nikookar, F. Hoeksema, "Adaptive Ad-hoc Free Band Wireless Communications", Project plan for AAF, Twente, 19 May 2004
[2] Li-Chun Wanga and Chung-Wei Wang, "Spectrum Handoff for Cognitive Radio Networks: Reactive-Sensing or Proactive-Sensing?", IPCCC 2008, pp 343-348.

[3] Amir Ghasemi and Elvino S. Sousa, "Opportunistic Spectrum Access in Fading Channels Through Collaborative Sensing", Journal of Communications, Vol. 2, No. 2, March 2007.

[4] Priyank Anand et.al, "Collaborative spectrum sensing in the presense of Byzantine attacks in cognitive radio networks", Second International Conference on Communication Systems and Networks (COMSNETS), Jan. 2010

[5] Uzma Siddique, Laeeq Ahmed, Habibulah Jamal, " Scattered spectrum sensing in cognitive radio network", www.techrepublic.com

[6] Won-Yeol Lee, Ian. F. Akyildiz, "Optimal spectrum sensing framework for cognitive radio networks", IEEE Wireless Comunication, Vol. 7, No. 10, October 2008.

[7] Edward Chu Yeow Peh et.al. "Optimization of cooperative sensing in cognitive radio networks: A sensing- throughput tradeoff view" , IEEE Transactions on vehicular technology, Vol. 58, No. 9, November 2009

[8] Sasirekha GVK, Jyotsna Bapat, "Optimal Number of Sensors in Energy Efficient Distributed Spectrum Sensing”, CogART, ISABEL, Rome, 2010,ISBN 978-1-4244-8131-6, DOI: 10.1109/ISABEL.2010.5702906

[9] www.ieee802.org/22/, "IEEE 802 LAN/MAN Standards Committee, 802.22 WG on WRANs (Wireless Regional Area Networks)"

[10] Hyoil Kim and Kang G Shin, “Efficient Discovery of Spectrum Sensing Opportunities with MAC-Layer Sensing in Cognitive Radio Networks", IEEE Transactions on Mobile Computing, Vol. 7, No.5, May 2008.

[11] Wei Zhang, Rajan K. Mallik, Khaled Ben Letaief, "Optimization of cooperative spectrum sensing with energy detection in cognitive radio networks',IEEE Transactions on Wireless Comunications, Vol. 8, no. 12, December 2009.

[12] Ian F. Akyildiz, Won-Yeol Lee, Kaushik R. ChowdhuryI.F. "CRAHNs: Cognitive radio ad hoc networks, Ad Hoc Networks". (2009), doi: 10.1016/ j.adhoc.2009.01.001

[13] Madhusudan Singh, Dhananjay Singh, "Impact and Performance of Mobility Models in Wireless Ad-hoc Networks", Fourth International Conference on Computer Sciences and Convergence Information Technology, 2009.

[14] Jari Nieminen, Riku Jäntti and, Lijun Qian, "Time synchronisation of cognitive radio networks", IEEE Globecom 2009

[15] Z.Chair and P.K. Varshney, "Optimal Data Fusion in Multiple Sensor Detection Systems", IEEE Transactions on Aerospace and electronic systems, Vol. AES-22, No.1 January 1986, Pages 98-101.

[16] Yunfei Chen, "Optimum Number of Secondary Users in Collaborative Spectrum Sensing Considering Resources Usage Efficiency", IEEE Communication Letters, Vol. 12, No. 12, December 2008.

[17] P.K. Varshney, Distributed Detection \& Data Fusion, Springer-Verlag, 1997.

[18] Chunhua Sun, Wei Chen and Khaled Ben Letaief , "Joint Scheduling and Cooperative Sensing in Cognitive Radios: A Game Theoretic Approach" IEEE WCNC proceedings 2009.

[19] Kristen Voyach, Padmini Pyapali and Anant Sahai, "Can we incentivize sensing in a light -handed way?”, IEEE DySPAN 2010.

[20] Netlogo: http://ccl.northwestern.edu/netlogo/

[21] Kun Zeng; Paweczak, P.; Cabric, D, “ Reputation-based cooperative spectrum sensing with trusted nodes assistance", Communications Letters, IEEE , Issue Date: March 2010 , Volume: 14 Issue:3 , Page: 226 -228 ISSN: 1089-7798

[22] Sina Maleki, Ashish Pandharipande, Geert Leus, “ Energy-efficient distrubuted spectrum sensing for cognitive sensor networks", IEEE Sensors Journal, Vol. 11, No. 3, March 2001

[23] Mansouri, N. Fathi, M, Simple counting rule for optimal data fusion, Conference on Control Applications, CCA 2003. Proceedings of 2003 IEEE , Vol. 2, June 2003, Pages: 1186- 1191,ISBN: 0-7803-7729-X 